\title{
A SUPERCONDUCTING 7T MULTIPOLE WIGGLER FOR THE BESSY II SYNCHROTRON RADIATION SOURCE*
}

\author{
D. Berger ${ }^{4}$, M. Fedurin ${ }^{2}$, M. Mezentsev², S. Mhaskar ${ }^{3}$, V. Shkaruba ${ }^{2}$, F. Schaefers ${ }^{1}$, M. Scheer ${ }^{1}$, E. Weihreter ${ }^{1}$ \\ ${ }^{1}$ BESSY, Einsteinstraße 15, 12489 Berlin, Germany, ${ }^{2}$ BINP, Acad. Lavrentiev \\ prospect 11, 630090 Novosibirsk, Russia, ${ }^{3}$ CAT, Indore 452 013, Madhya Pradesh, \\ India, ${ }^{4}$ Hahn-Meitner-Institut, Glienicker Straße 100, 14109 Berlin, Germany
}

\begin{abstract}
To generate hard X-ray beams for residual stress analysis and for magnetic scattering using the BESSY II ring, a $7 \mathrm{~T}$ wiggler with 17 poles is under development. The wiggler is designed for a critical energy of $16.8 \mathrm{keV}$ with a $13 \mathrm{~mm}$ vertical free aperture and an inner chamber on a temperature of $20 \mathrm{~K}$. Essential aspects of the conceptual magnet layout are discussed, e.g. coil and yoke geometry, and vacuum chamber design. A prototype structure with 7 poles has already been built to verify the technical layout of the wiggler. First experimental results for the prototype magnet are presented, demonstrating that a maximum field of $7.3 \mathrm{~T}$ can be obtained with the present design.
\end{abstract}

\section{INTRODUCTION}

The BESSY II storage ring is operating as a high brilliance Synchrotron radiation (SR) source for the VUV and soft X-ray spectral range. There is however an increasing demand for hard X-ray beams for structural biology, solid state physics and industrial applications. In collaboration with the Hahn-Meitner-Institut dedicated beamlines are under development for magnetic scattering and for residual stress analysis. Particularly for the latter application a high flux and a deep penetration $(1 \mu \mathrm{m}-1$ $\mathrm{mm}$ ) of the photon beam into the (most often technical) samples are mandatory, with a photon flux in the range of $10^{10} \mathrm{~s}^{-1}$ at $60 \mathrm{keV}$ in a $0.1 \%$ bandwidth through an aperture of $0.5 \times 0.5 \mathrm{~mm}^{2}$ in a distance of $30 \mathrm{~m}$ for a 1.9 $\mathrm{GeV} / 200 \mathrm{~mA}$ beam. In Fig. 1 the photon flux of the superconducting $7 \mathrm{~T}$ multipole wiggler (MPW) is compared to other insertion devices at the BESSY II storage ring. At higher energies beyond $10 \mathrm{keV}$ the photon flux of the MPW is superior to the flux offered by other insertion devices. Thanks to the high flexibility of the BESSY II lattice with alternating high and low beta straight sections[1] such high field devices can be implemented into the ring without severe beam dynamical implications. The essential parameters of the MPW are summarized in Table 1.

\section{CONCEPTUAL DESIGN}

As shown in Figs. 2 and 3 the concept of the wiggler is based on 17 iron poles that are excited by superconducting NbTi-coils of racetrack shape. A configuration with 2 endpoles of $1 / 4$ and $3 / 4$ of the full field

\footnotetext{
*work supported by BMBF / Germany (HGF Strategiefond)
}

Table 1: Multipol wiggler design parameters

\begin{tabular}{|l|r|}
\hline critical energy @ $1,9 \mathrm{GeV}$ & $16.8 \mathrm{keV}$ \\
\hline max. field on axis & $7.0 \mathrm{~T}$ \\
\hline max. field on coils & $8.1 \mathrm{~T}$ \\
\hline periode length & $148 \mathrm{~mm}$ \\
\hline number of poles & 17 \\
\hline horiz. beam aperture & $110 \mathrm{~mm}$ \\
\hline vert. beam aperture & $13 \mathrm{~mm}$ \\
\hline magnetic (iron) gap & $19 \mathrm{~mm}$ \\
\hline stored magnetic energy & $450 \mathrm{~kJ}$ \\
\hline total radiation power $(1.9 \mathrm{GeV}, 500 \mathrm{~mA})$ & $56 \mathrm{~kW}$ \\
\hline
\end{tabular}

strength has been chosen. Therefore the beam orbit oscillates horizontally around the vertical symmetry plane of the magnet with the advantage that most of the influence of the integral sextupole term on the beam will cancel.

Each pole is excited by two individual coil sections using two separate power supplies (exept $1 / 4$ endpoles) [1] to allow the currents to be chosen near the critical value of the superconducting wire depending on the maximum field at the respective coil section. For $7 \mathrm{~T}$ the currents reach $75 \%$ of the short sample limit of the superconducting wire. A round superconducting wire of

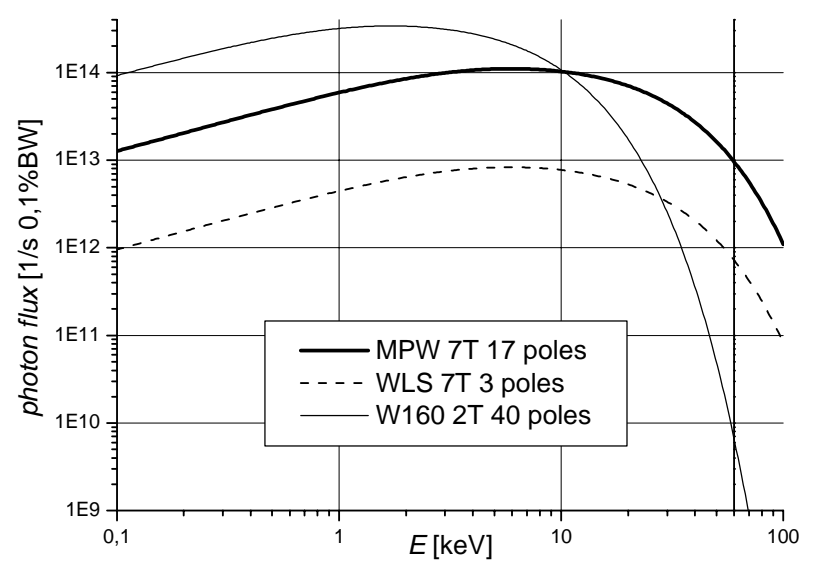

Figure 1: Spectral photon flux into an aperture of $1 \times 1$ $\mathrm{cm}^{2}$ at $10 \mathrm{~m}$ distance from the middle of the source for the superconducting multipole wiggler ( $7 \mathrm{~T}, 17$ poles) compared with other X-ray sources at BESSY II such as a wavelength shifter ( $7 \mathrm{~T}, 3$ poles) and a permanent magnet wiggler W160 ( $2 \mathrm{~T}, 40$ poles) 
$0.9 \mathrm{~mm}$ diameter (8910 filaments of $6 \mu \mathrm{m}$ size) with a $\mathrm{NbTi} / \mathrm{Cu}$ ratio of 1:1.3 and a critical current of $380 \mathrm{~A}$ at $7 \mathrm{~T}$ will be used. Much effort was made to develop a combined welding and compression technique to reduce the residual resistance of the 68 connections between the coils with the result of a resistance of $\approx 10^{-11} \mathrm{Ohm}$ per connection.

A vertical cut through the wiggler is shown in Fig. 3. The iron yoke made from low carbon ARMCO steel returns the magnetic flux from pole to pole and supports the coils. Additional iron plates are used to return the stray field at both ends of the wiggler. A $10 \mathrm{~mm}$ gap formed by stainless steel plates is included in the magnetic circuit below and above the coils homogenising the field distribution in the gap region thus reducing the magnetic force on the beam chamber.

The radiation of the wiggler will be emitted in a horizontal angle of $+/-25.6 \mathrm{mrad}$ (see Fig. 4) giving a total power of $22.4 \mathrm{~kW}$ under nominal user operation conditions $(1.9 \mathrm{GeV}, 200 \mathrm{~mA})$. The wiggler beam chamber together with the vacuum chamber downstream of the wiggler, however, will be designed for a power

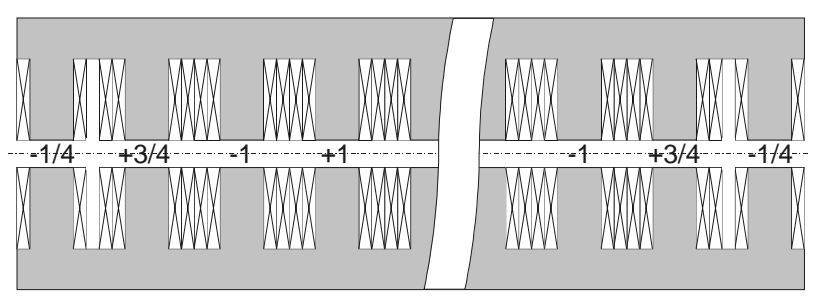

Fig. 2: Schematic configuration of the wiggler with 13 full poles (+/- 1) and 2 endpoles (-1/4 and 3/4) on each side

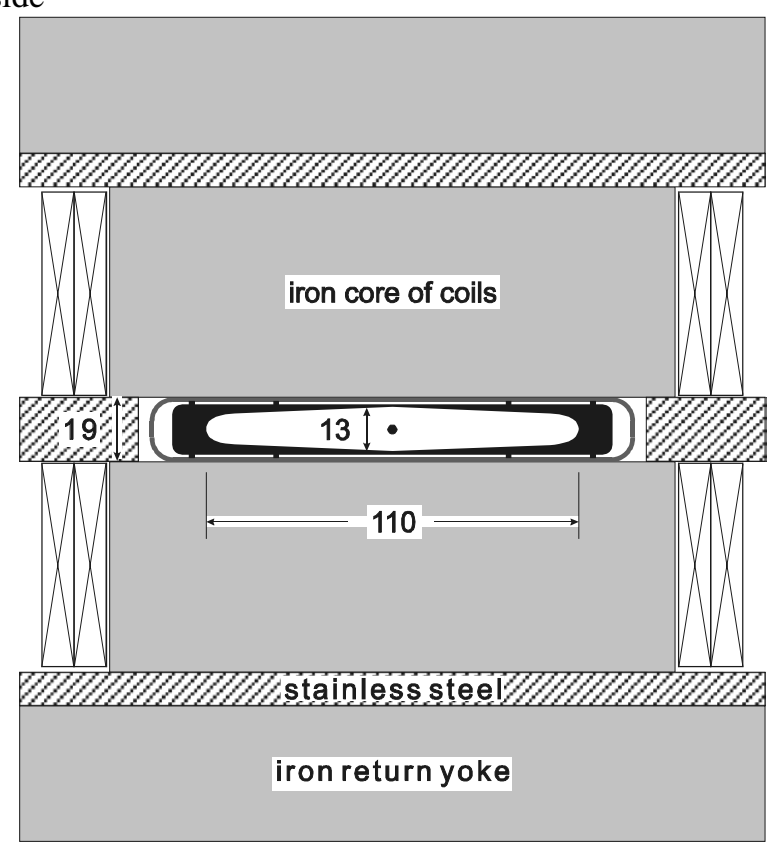

Fig. 3: Vertical cross-section of the wiggler with the vacuum chamber (@ 4.2K) and the inner radiation shield (@20K)

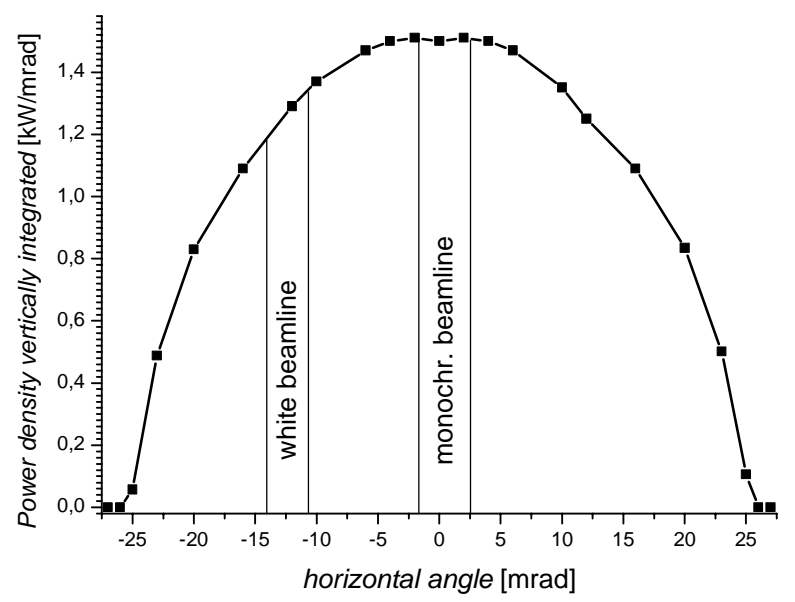

Figure 4: Horizontal power distribution of the $7 \mathrm{~T}$ multipole wiggler at $1.9 \mathrm{GeV} / 500 \mathrm{~mA}$, total power 56 $\mathrm{kW}$

level of $56 \mathrm{~kW}$, i.e. maximum power density of $2 * 10^{9}$ $\mathrm{W} / \mathrm{m}^{2}$, to allow for a future upgrade of the beam current to $500 \mathrm{~mA}$. Therefore great care must be taken in the design of these chambers.

To minimise the heat load on the cold vacuum chamber $(4.2 \mathrm{~K})$ of the wiggler a radiation shield made of copper will be placed around the beam as shown in Fig. 3, which is connected to the $20 \mathrm{~K}$ shield of the cryostat. This shield protects the cold vacuum chamber from power exposures due to resistive wall power losses, low energy SR emitted vertically by the wiggler, SR emitted upstream of the wiggler in the fringe field of the dipole magnet, and from the radiation scattered backward from the first absorber after the wiggler.

To operate the MPW at $4.2 \mathrm{~K}$ the magnet will be inserted into a liquid helium bath cryostat. Two radiation screens at $20 \mathrm{~K}$ and $60 \mathrm{~K}$ and high $\mathrm{T}_{\mathrm{C}}$ current leads together with many layers of superisolation help to keep the heat flux from outside at a low level. Four commercial cryo coolers will be used to recondense the gas and to cool the shields with a total cooling capacity of $2 \mathrm{~W}$ at $4.2 \mathrm{~K}$ (and $140 \mathrm{~W} / 25 \mathrm{~W}$ at $60 \mathrm{~K} / 20 \mathrm{~K}$ respectively), allowing for a closed loop operation mode without the need for regular LHe refillings.

\section{PROTOTYPE TEST MEASUREMENTS}

The 17 pole wiggler will be manufactured at BINP in the second half of 2001. In a first step a 7 pole prototype wiggler (see Fig. 5) has been built and tested in a bath cryostat to verify the proposed magnet design and to demonstrate that the specified field can be reached in order to allow for a reliable long term operation at $7 \mathrm{~T}$.

To measure the magnetic field distribution in the median plane of the wiggler, 5 Hall probes were located on a carriage which can be moved inside the cryostat along the wiggler axis by a stepping motor. The Hall probes were calibrated before at $4.2 \mathrm{~K}$ using an NMR probe, and the coil currents were measured by high precision DC current 


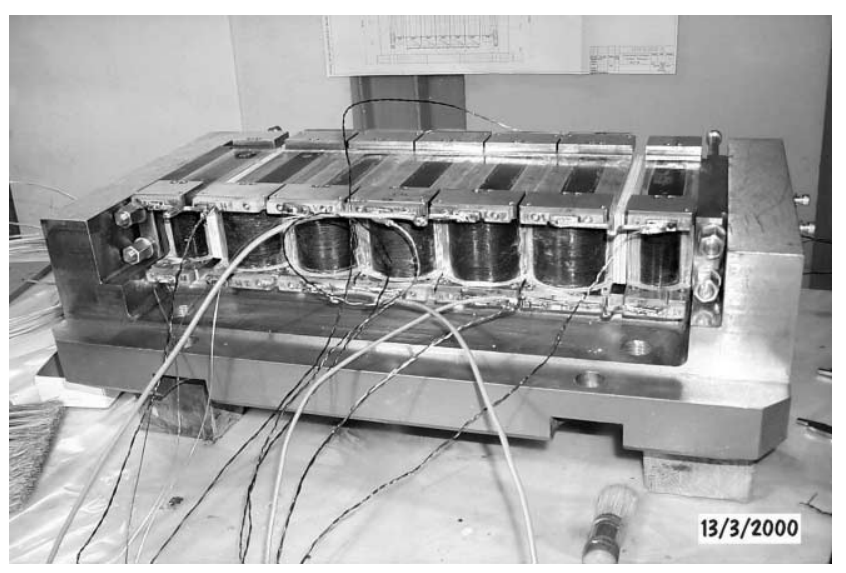

Fig. 5: Lower half of wiggler prototype with 3 main poles and 2 sidepoles on each side

transformers. As an example Fig. 6 shows the measured field distribution along the axis in comparison with a 3D field calculation where a known systematic error of the numerical code has been taken into account.

In a first series of test measurements (see left part of Fig. 7) a maximum field of $7.1 \mathrm{~T}$ was reached after 20 quenches. Most of the quenches occured in the inner coil sections of the central main pole which are subject to the highest fields. The quenches were evenly distributed between the upper and lower poles. As during these quenches the currents in the coils were still significantly below the critical value of the superconducting wire, the quenches could be attributed to small wire motions due to insufficient pre-stressing of the coils.

In a second test the pre-stressing force was increased by a factor of 1.3 using additional tension screws. Under these conditions a maximum field of $7.3 \mathrm{~T}$ could be reached after 16 quenches as shown in the right part of Fig. 7. This underlines the importance of an adequate prestressing system.

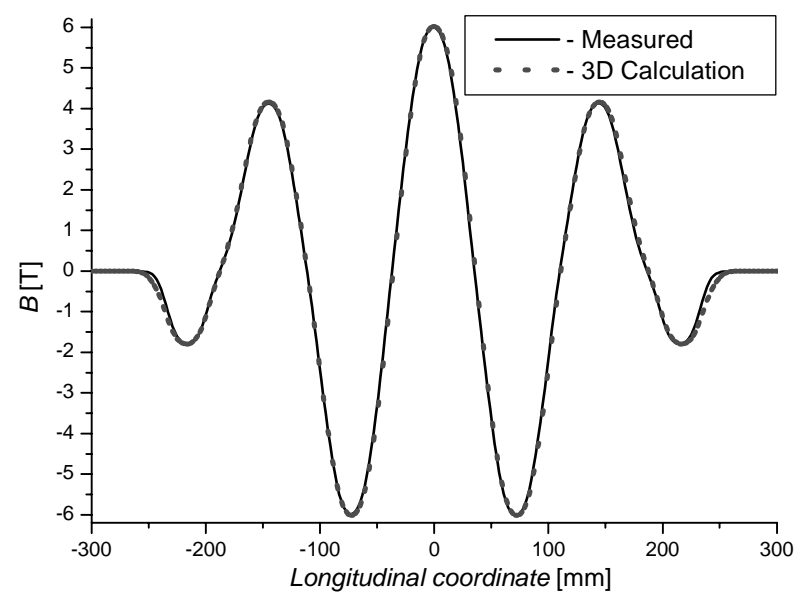

Fig. 6: Longitudinal field distribution of the prototype

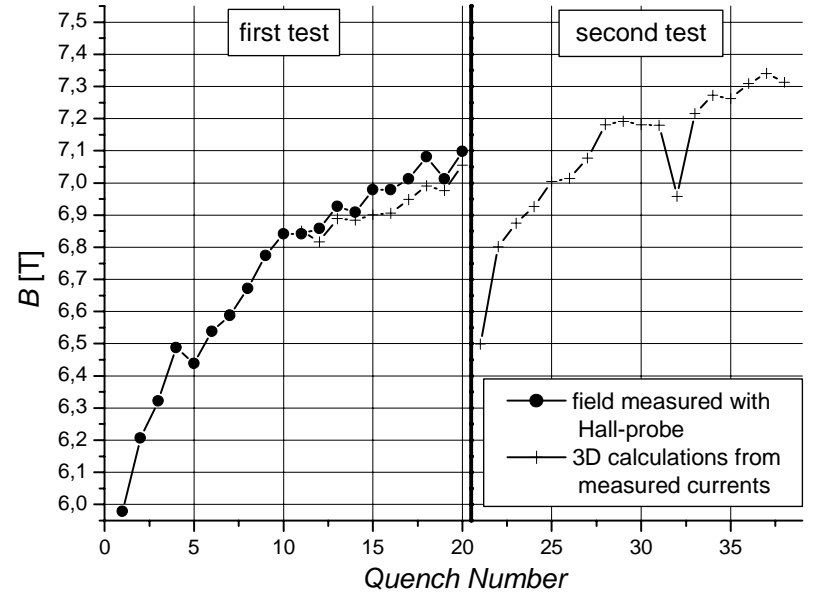

Fig.7: Quench history of prototype, maximum reached field is $7.3 \mathrm{~T}$

\section{CONCLUSION AND NEXT STEPS}

The quench tests of the prototype magnet have shown that a maximum field of $7.3 \mathrm{~T}$ can be obtained based on the present wiggler design, slightly above the design goal of 7.0 T. To improve the safety margin for operation of the final 17 pole wiggler the pre-stressing system will be modified together with some minor parameter changes and improvements in coil fabrication technology. A third prototype test will be performed soon to verify the modifications with the aim to have the final wiggler ready for tests early next year.

\section{REFERENCES}

[1] G.A. Voss, DESY Internal Report 1998

[2] M. Scheer, G. Wüstefeld, Proc. of the Europ. Part. Acc. Conf. 1992, Vol. 1, p. 676

\section{Aknowledgments}

The authors would like to thank G. A. Voss, S.L. Wipf and S. Wolf from DESY for many helpful discussions. 